\title{
Factors of the Design of the Fretting Phenomenon in the Development of Health Saving Shoes
}

\author{
Sergey P. ALEXANDROV \\ Ph.D. \\ Professor \\ Head of the department \\ Leather, Fur and Leather Products Technologies Department \\ Moscow State University of Technology and Management K.G. Razumovsky (FKU) \\ 73, Zemlyanoi Val Str., Moscow, 109004, Russia \\ mor-galina@yandex.ru
}

\section{Tatyana V. ZHUKOVSKAYA}

Ph.D.

Associate Professor

Garment and Footwear Design Department

Faculty of Design and Software Engineering

Kazan National Research Technological University

68, Karl Marx Str., Kazan, 420015, Russia

utrennyaya@mail.ru

\begin{abstract}
When designing the health-protecting footwear, the special focus is on the ergonomic features that characterize the level of matching of the footwear to the foot. They are of primary concern when creating the casual shoes, as well as the footwear for children, the elderly people and the pregnant women. When considering the interference of the footwear with the foot in the process of bedding or matching its inside to the shape of the human foot, one of the understudied phenomena that occurs during the process of walking is the fretting phenomenon, which leads to the appearance of roughness and calluses on the sections of the foot that contact with the inner surface of the footwear, destroys the material of the lining and the insole, as well as the product worn on the foot. In order to develop a calculation method of the fretting phenomenon in a pair of the ball part of the foot and insole, a model that includes system-forming elements, structure charts, fixed parameters, and control variables is proposed, which makes it possible to model study of the wear problem of movable contacting pairs and prevents its negative consequences.
\end{abstract}

Keywords: health saving shoes; ergonomic properties; fretting phenomenon; absorption; model study.

\section{Introduction}

In the problem of improving the quality of shoes, the issues of improving its ergonomic properties, because human health and working efficiency depend on them, take a special place. They are of special importance in the design of casual shoes, as well as shoes for children, the elderly people and the pregnant women (Rodgers, 1995; Ren-fei, Zhong-liang, 2006; Hessert, Vyas, Leach, 2005; Praet, Louwerens, 2003). Ergonomic indicators characterize the degree of adjustment of a product to a person and are based on the ergonomic properties of the "person-product-environment" system. The issue of improving the comfort of shoes and eliminating its negative impact on the foot is one of the main issues in terms of ergonomic characteristics. From this point of view, one of the littlestudied phenomena that occur in a pair of foot-shoe during walking is the fretting phenomenon. It 
causes violations of conditions of the shoe fitness, leads to the appearance of roughness in the areas of the foot that are in contact with the internal surface of the shoe, destroys the material of shoe lining and insole, as well as the product worn on the foot. There is the fretting on the surfaces of tightly pressed bodies at small relative shifts, while the interaction products remain in the section of moving contact.

The purpose of this study is to identify the factors of the fretting phenomenon model in the pair of the ball part of the foot and the insole in order to study the wear of movable contacting pairs and to prevent the negative consequences of this phenomenon.

\section{Methods}

One of the active sections of the fretting phenomenon in a pair of foot-shoe takes place in the ball part, where there is the movement of the heads of metatarsal bones and, subsequently, the plantar aponeurosis with fascia along the upper surface of the insole.

In order to develop a calculation method or an experimental study of the fretting process and its consequences, it is necessary to create an object model that includes system-forming elements, structure charts, fixed parameters and control variables. The model should also contain evaluation output submodels (criteria), functional relations - forward and backward.

The longitudinal arch is an arch that is capable of resiliently spread apart under the load action, comprises of external and internal longitudinal arches. The latter whilst the rolling motion of the foot from the seat of the heel is the first to engage with the supporting surface through fabric connections and to transmit the end reaction in the form of a spread load on the external longitudinal arch, which can be reduced to the moment in the frontal plane and to the force in the sagittal plane.

The opposing torque in the first and fifth heads of metatarsus balances the moment that influences the lateral stability of the foot and its impact on the spreading apart of the arch is negligible.

The reaction of the supporting surface acting on the internal arch will indirectly affect the end reaction of the longitudinal arch of the foot, which is sufficiently determined by the computationalexperimental method using modern hardware-software measurement complexes that enables to consider in the design model of spreading apart of the longitudinal arch the impact of the internal arch.

As a result, the longitudinal arch model can reasonably be reduced to a planar representation.

In the process of studying the fretting phenomenon that occurs during the interaction of the ball part of the foot and the insole, the determining value is the shift function and the force in the area of moving contact. To locate the unknown quantities, the hinged-rod mechanism with geometrics and force parameters corresponding to the subject of research could be a replacement model.

The junction of the heads of the tibia and talus bones forms the top hinge of the model; the heel rod models a double-section chain from talus and heel bones; the second rod replaces the chain that consists of the talus, navicular, cuneiform, metatarsal and heads of the metatarsal bones. Clear-cut ideas of longitudinal arch models were made in earlier works as well (Gefen, 2002; Alexandrov, Parshina, 1989; Mruz, 2004).

The connection of the calcaneal tuber with the supporting surface is modeled by an immovable hinged support with the possibility of rotation only; the connection of the heads of metatarsal bones with the supporting surface is represented by a movable hinged support with the possibility of rotation and longitudinal axial movement along the supporting surface. The calcaneal tuber is connected to the heads of the metatarsal bones with elastic tendons (plantar aponeurosis) - returning the arch spread apart under load to its initial position when the load is removed, which are modeled 
by an elastic tie located between the horizontally arranged hinges of the model's replacement mechanism.

For refine calculations, when forming a model of the longitudinal arch, it should be taken into account the deformity of the joints in the chain of synostosis in the first place, from the talus to the metatarsal bones, where the joints are placed in both sequential and parallel order. The general deformation can be determined through a generalized index of stiffness calculated for series-parallel connection.

In statically reel feet with evident longitudinal flat feet, the arch apparently does not spread apart and, subsequently, the elastic tie of the model is not distorted. The human body loses its natural shock absorber. The stiffness coefficient of the arch in terms of the vertical component will make (1):

$$
\mathrm{C}=\frac{m g}{k \Delta}
$$

where $\Delta$ - value of the horizontal spreading apart of the foot during the load action $\mathrm{mg}$;

$\mathrm{k}$ - coefficient of conversion of the value into the vertical shift, equals to 0,4 .

In order for the body and vital organs of a person not to go through the overexertion in case of static deformation of the feet, it is necessary to decrease the stiffness of the system of the shoe bottom, by sequential addition of the stiffness of the arch $\mathrm{C}$.

The spreading apart of the longitudinal arch and the replacing it hinged-rod model is carried out from the beginning of the contact of the ball part of the shoe with the support until the moment of separation of the heel area of the shoe from the support, which amounts to about a third part of the contact of the shod foot with the support. At normal walking pace $(\sim 5 \mathrm{~km} / \mathrm{h})$, the time of spreading apart of the arch of the foot is $\sim 0.4 \mathrm{~s}$. Given that the average value of the spreading apart of the arch is defined as $3 \mathrm{~mm}$, then the speed of spreading apart takes $\mathrm{V} \sim 7.5 \mathrm{~mm} / \mathrm{s}$.

The data source on the elements of the replacement model, their siting and measures could be generally accepted anatomic studies of the foot, presented in the medical books (Perepyolkin, 2013).

With knowledge of load value acting through the ankle joint on the model of the foot presented for the position without a heel and with a heel (Fig. 1), we perform a force study.

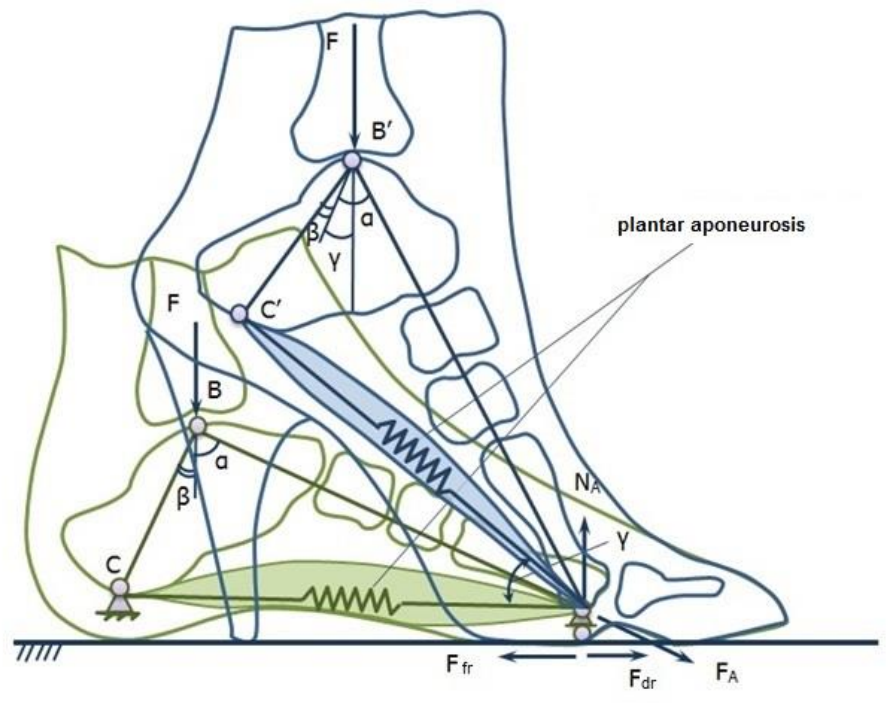




\section{Figure 1. Construction of longitudinal arch of the foot and its model}

Let's consider the position of the model corresponding to the raise of the seat of the heel of the foot to various pitches of the heel. Using the method of kinetic-static analysis of the hinged-rod mechanisms, we find the values of the load force $\mathrm{F}_{\mathrm{A}}$ acting on the rod $\mathrm{AB}$, using the formula (2):

$$
F_{A}=F \times \frac{\sin (\beta+\alpha)}{\sin (\alpha+\beta)}
$$

Next we find the normal force $\mathrm{N}_{\mathrm{A}}$ and the force of spreading out the $\operatorname{arch} \mathrm{F}_{\mathrm{dr}}(3)$ :

$$
\begin{aligned}
& N_{A}=F_{A} \times \cos (\alpha-\gamma) \\
& F_{d r}=F_{A} \times \sin (\alpha-\gamma)
\end{aligned}
$$

The conducted measurements of the friction coefficients of pairs of materials of foot with a sock and without a sock with different types of insoles showed that the friction coefficient is in the range of $0.1 \div 0.5$ (Alexandrov, Zhukovskaya, 2013). Therefore, the friction forces were defined at extreme values of the friction coefficients, which made it possible to estimate the field of their permissible variations (4).

$$
\begin{aligned}
& F_{f r}^{\mathrm{H}}=N \times \mu_{\mathrm{H}} ; \mu_{\mathrm{H}}=0,1 \\
& F_{f r}^{\mathrm{B}}=N \times \mu_{\mathrm{B}} ; \mu_{\mathrm{B}}=0,5
\end{aligned}
$$

The range of sizes of the angle of ascent of the foot was assumed from $0^{\circ}$ to $45^{\circ}$, the last value corresponds to the pitch of the heel $\mathrm{h}=100 \mathrm{~mm}$. Thus, calculated values of $\mathrm{F}_{\mathrm{dr}}, F_{f r}^{\mathrm{H}}, F_{f r}^{\mathrm{B}}$ are presented in Fig. 2.

The curve $F_{f r}^{\mathrm{B}}(\mu=0.5)$ intersects the line $\mathrm{F}_{\mathrm{dr}}$ at the point $\mathrm{L}$, which shows the impossibility of spreading apart the arch at an angle of ascent of the foot starting from $24^{\circ}$, since the spreading apart force $F_{\mathrm{dr}}$ is completely compensated by the friction force $F_{f r}^{\mathrm{B}}$. At minimum values of the friction coefficient $\mu=0.1$, which is realized in a pair of kapron sock - leather for insoles, the curves $\mathrm{F}_{\mathrm{dr}}$ and $F_{f r}^{\mathrm{H}}$ intersect at the point $\mathrm{K}$, corresponding to an angle of ascent of the foot $43^{\circ}$ or the pitch of the heel $95 \mathrm{~mm}$. At this point, the spreading apart the longitudinal arch force is entirely neutralized by the friction force $F_{f r}^{\mathrm{H}}$ at $\mu=0.1$ and the natural shock absorption disappears. 


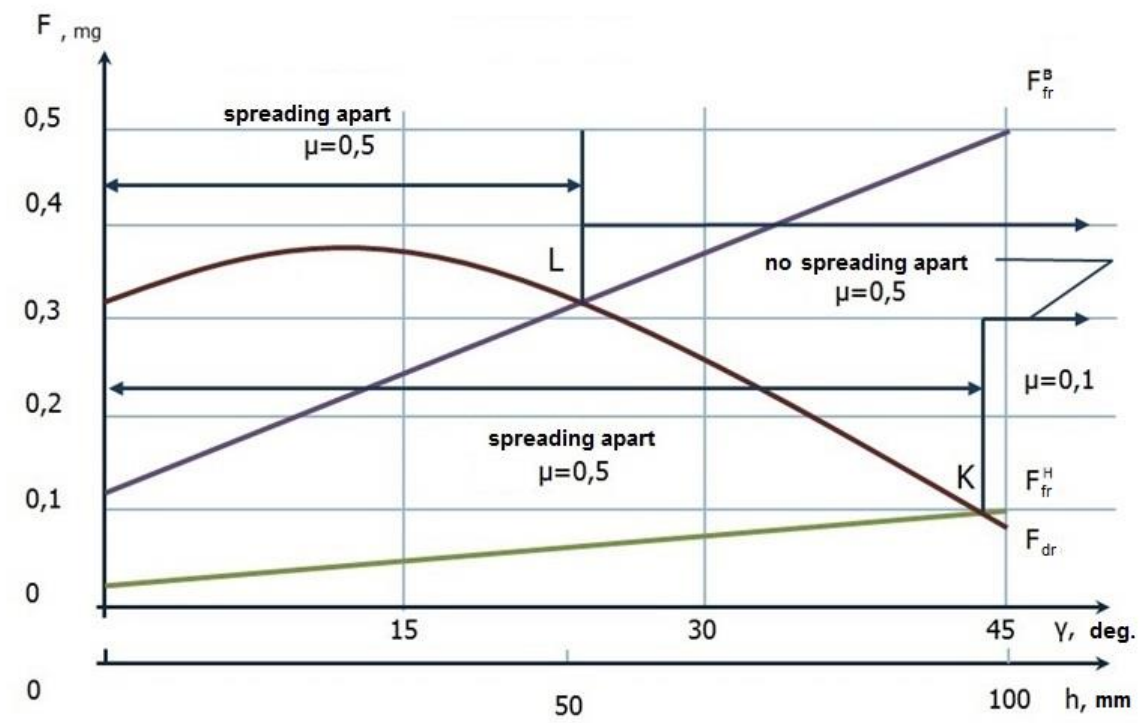

\section{Figure 2. Graphs of variation $F_{f r}^{\mathrm{B}}, F_{f r}^{\mathrm{H}}, F_{\mathrm{dr}}$ as a function of an angle of ascent of the foot and the pitch of the heel}

The force spreading apart a plantar aponeurosis (in the model, an elastic ligament of horizontal hinges) is equal to the difference between the driving force and the friction force $\mathrm{F}_{\mathrm{sa}}=\mathrm{F}_{\mathrm{dr}}-\mathrm{F}_{\mathrm{fr}}$

As can be seen from the graph (Fig. 2), this difference of the force $F_{\text {sa }}$ until the ascent of the foot to an angle of $15^{\circ}$ remains approximately constant and at $(\mu=0.1)$ is $\sim 0.3 \mathrm{~F}$. The maximum value of the force $\mathrm{F}$ acting on the ankle joint (upper hinge $\mathrm{B}$ of the model) in phase of full support on the foot, reaches the value $\mathrm{mg}$ at the spreading apart of the arch by $3 \mathrm{~mm}$. In the example cited of the female foot, the stiffness coefficient of the plantar aponeurosis (elastic tie) will make $\mathrm{C}=0.3 \mathrm{~F} / \Delta=$ $0.3 \times \mathrm{mg} / \Delta \approx 70 \mathrm{~N} / \mathrm{mm}$.

It can be observed that with the raise of the angle of ascent of the foot, not only the marked points of intersection $\mathrm{F}_{\mathrm{sa}}$ with $F_{f r}^{\mathrm{H}}$ and $F_{f r}^{\mathrm{B}}$ are dangerous, that show the limiting values of the raise at which the spreading apart of the arch is equal to zero. If we analyze the option of spreading apart the arch of the foot generated by nature, then with a man walking without a heel, which occurred for more than 2 million years, the force delivered on the plantar aponeurosis, responsible for the shock absorption of the human body, evaluates as $\Delta \mathrm{F}=\mathrm{F}_{\mathrm{sa}}-F_{f r}^{\mathrm{B}}$ at $\gamma=0$.

At the angle of ascent of the foot, where the difference between $F_{F R}$ and $F_{F R}$ will be twice as little as at the angle $\gamma=0$ and such a force will exert the spreading apart of the arch not $3 \mathrm{~mm}$, as formed by nature, but $\Delta_{1}=0.1 \cdot \mathrm{F} / 0.067 \cdot \mathrm{F}=1.5 \mathrm{~mm}$, i.e., the level of shock absorption of the human body will be also diminished by 2 times, which will have a negative effect on his musculo-skeletal system. The reduction of the amplitude of the reciprocal movement of the ball part of the foot on the surface of insole will also affect the process of fretting wear problem. 


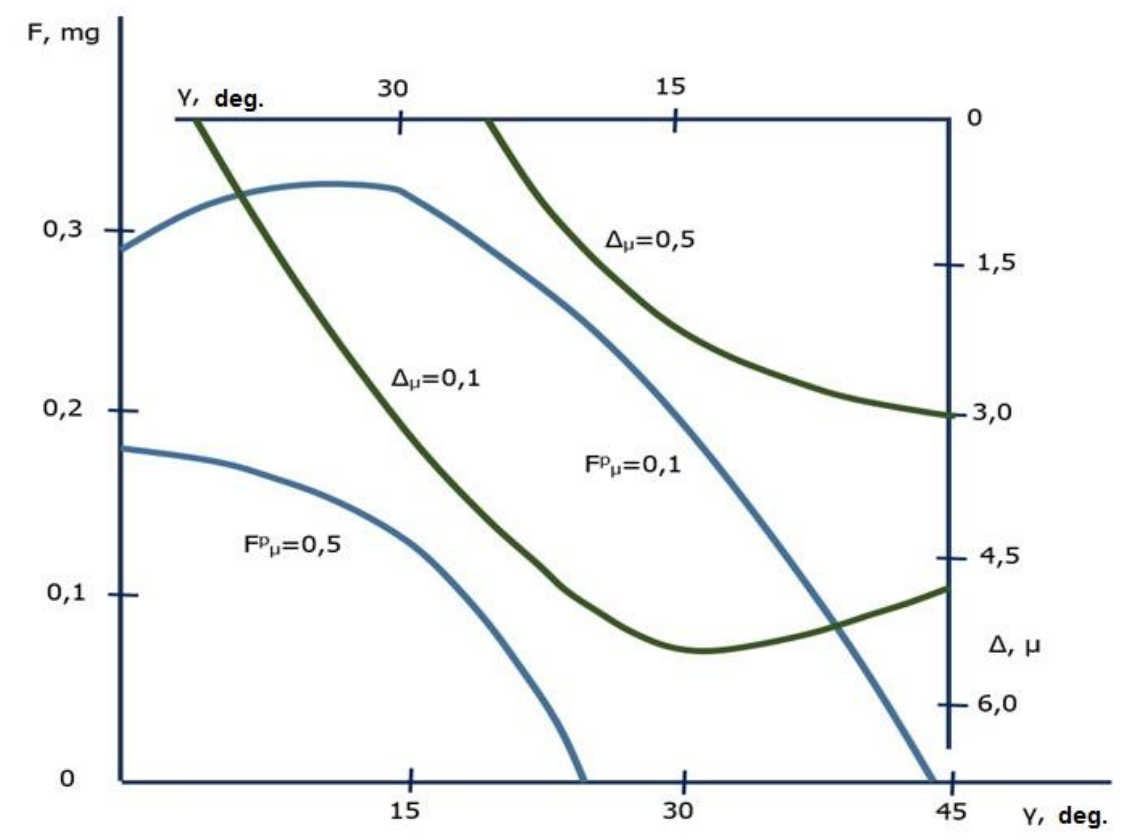

Figure 3. Dependency of spreading apart of the arch of the foot forces and elongations from the friction coefficients and the angle of ascent of the foot

Figure 3 shows the dependences of the spreading apart of the arch of the foot forces $\mathrm{F}^{\mathrm{p}}$ at $\mu=0.5$ and $\mathrm{F}^{\mathrm{p}}$ at $\mu=0.1$ from the angle of ascent of the foot $\gamma$, as well as the dependences of the elongation of the arch $\Delta$ at $\mu=0.5$ and $\Delta$ at $\mu=0.1$ (determined by the opposite coordinates of the graph) from the angle of ascent of the foot.

We suggest carrying out an experimental-analytical determination of the matrix of the elementary pressures on the contact surface of the foot-insole and the total forces at the selected areas of the model, in phase of the full foot support, on the hardware-software complex DiaSole-M-Scan (Smirnova, Iuldashev, Ioffe, Ioffe, 2010). Before carrying out the research, an insole with built-in primary measuring transducers (the pressure sensors) was inserted into the shoes of a preset size and a heel height. The subject put on shoes in the seated position, the outside wires of the measuring insole were connected to the mobile processor. The subject rose from a chair, leaning on both legs, and performed few steps in the traditional pace. In phase of the full foot support, information from the sensors, reflecting the "point" load distribution on the insole, was highlighted in the software module, formatted into a numerical pressure matrix (Fig. 4) and into the spatial pressure diagrams.

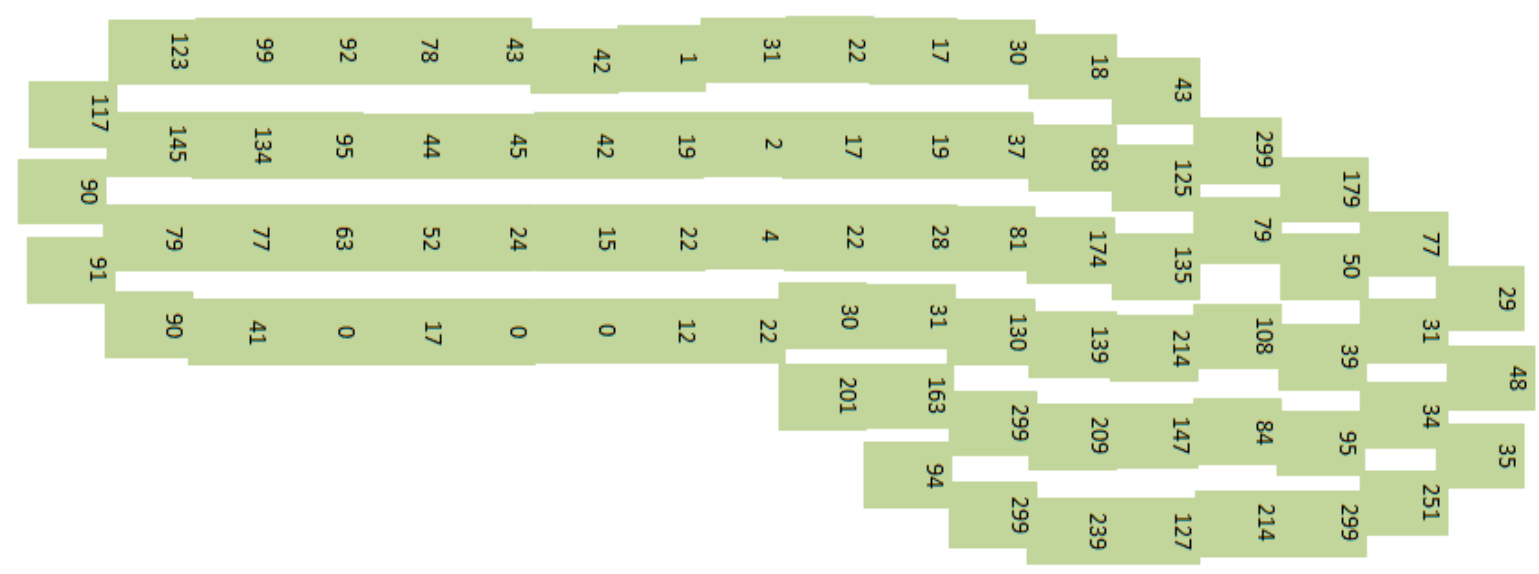

Figure 4. The distribution of the pressures on the insoles of high-heeled shoes in phase of the full foot support (the figures are provided in the dimension $\times 10 \mathrm{~g} / \mathrm{cm}^{2}$ ) 
The pressure matrix suggests that in phase of the full foot support, the constructive raise of the toe and ball part of the bottom of high-heeled shoes under the load transforms into a horizontal plane, which in the model representation is reflected as a movement of the support hinge (Figure 1) along a horizontal line.

Using the pressure matrix, its digital data is translated, with a known area of the sensors, into the dimension of forces, still three groups of unidirectional forces are generated, one on each selected area of the foot, having an inherent angle of inclination, normal to which the vectors of these forces are placed. The force matrix obtained from the pressure matrix, as well as the latter, has the longitudinal axis, indicated OX, passing through all the selected areas. The introduction of the single longitudinal axis is caused by the transition from a spatial object of the foot-insole pair to a flat model. On each area, the transversal axis is drawn - OY of the boundary lines of the adjoining areas. The centers of the elementary efforts are placed on both sides of the OX axis on the lines perpendicular to it.

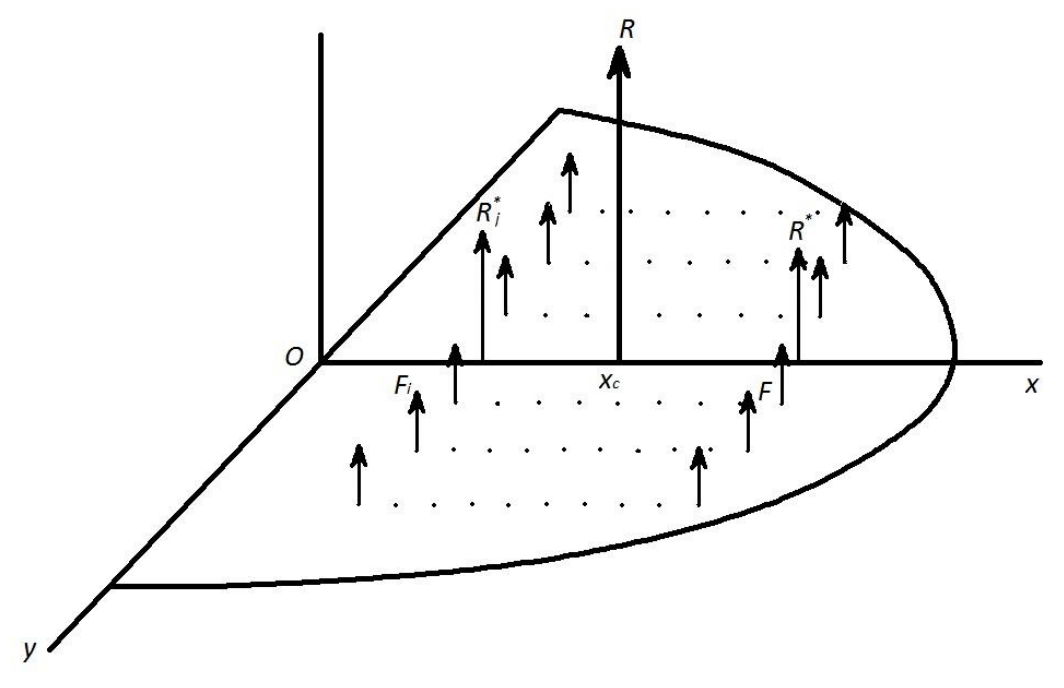

\section{Figure 5. The reduction of the elementary forces $\mathrm{Fi}$ to the resultant $\mathrm{R}$ on the toe and ball part}

For the force analysis of the interaction of the foot and the insole, it is necessary to define, in addition to the resultant force on each area, its coordinates.

On the toe and ball area, taken as an example (Figure 5), we convert the set of forces Fi acting on the XOY plane into a limited number of efforts equal to the number of perpendiculars to the OX axis, on which the centers of elementary forces are located. We will install the location of the new force vectors at the intersection of the perpendiculars with the axis of symmetry OX, on which the areas of the model are placed. The elementary forces with the centers of the application on the perpendicular are replaced by the resultant with the center at the intersection with the OX axis and, given that the resultant of the parallel forces in absolute value is equal to the sum of the components, we write down its module

$$
\mathrm{R}^{*}=\sum \mathrm{F}_{\mathrm{i}}=\sum \mathrm{p}_{\mathrm{i}} \mathrm{A}
$$

where $\mathrm{p}_{\mathrm{i}}$ - the pressure measured by the sensor; $\mathrm{A}$ - the area of the sensor.

The longitudinal axis OX divides the matrix of each area into two to some extent symmetrical sides. The elementary forces, located on these sides create moments in relation to the OX axis that are sufficiently close in value and oppositely directed, which promotes the cancellation of the moments. The arising minor difference of the moments, aimed at the torsion of the insole about the longitudinal axis, in view of the rigidity of the insole and the bottom of the shoe related to it, 
counterbalances the excess torque, which makes it possible to disregard it within the defined problem.

The resultant $\mathrm{R}$ (more correct - the resultant vector) of all forces applied at the selected area passes through the point $\mathrm{Xc}$ - the center of parallel forces located on the longitudinal axis OX. The module of the resultant $\mathrm{R}=\sum \mathrm{R} *_{\mathrm{j}}$, the direction - perpendicularly to the OX axis (Figure 5).

Applying the Varignon theorem, we find the point Xc, writing down the moments of the new forces $\mathrm{R}^{*}{ }_{\mathrm{j}}$ and the resultant $\mathrm{R}$ in relation to the transversal axis $\mathrm{OY}$

$$
\mathrm{m}_{\mathrm{y}}(\mathrm{R})=\sum \mathrm{m}_{\mathrm{y}}\left(\mathrm{R}^{*}{ }_{\mathrm{j}}\right)
$$

Revealing the moments, we obtain:

$$
\mathrm{RXc}=\sum \mathrm{R}_{\mathrm{j}} \mathrm{X}_{\mathrm{j}}
$$

from where the $\mathrm{Xc}$ coordinate is determined, the second coordinate $\mathrm{Yc}=0$, which enables us to identify more precisely the geometrical parameters of the model.

In a similar way are determined the total forces and their coordinates of the centers of the application at other selected areas of the foot. For all the elements in contact with the areas of the supporting broken line, the substitution of the operation of the union is done by the normal reaction and the frictional force, the reactions in the coupler pins are determined by the forces that bring in the neighboring elements to a balanced condition.

Hence, during the separation of the longitudinal and the transverse arches of the foot, the lateral surfaces of the toe and ball part of the foot and its back area (excepting the upper zone, discontiguous with the toes) come into the power contact with the tightened shoe upper, which produces the retarding action, increasing with the advancement of the forefoot. The action force of the shoe upper on the toe and ball part of the foot, arising during the separation of the arch acts as an additional braking of the forefoot, and, in case of the weakened plantar aponeurosis prevents its plastic extension.

Consequently, in order to provide the immobility of the heel of the foot in relation to the surface of the insole in phase of the full support, the heel area of the insole should be specially treated or the structural changes should be introduced to increase the friction ratio $\mu=0.7-1.0$. In phase of the foot transfer, when the pressure of the plantar part of the foot on the insole falls to zero and, consequently, the frictional force in this pair disappears, the force of the distended aponeurosis, that overcame the frictional force of the backing of the upper and the toe and ball area of the foot in the stocking, will return the forefoot to the initial position of the unexposed arch of the foot.

\section{Results}

Thus, in the framework of problem solving of creation of health saving shoes, in this study the necessary conditions of the comfortability of the walking in the high-heel shoes are outlined: no slip of the entire foot on the insole surface; the elastic displacement of the waist and toe and ball part of the foot on the insole surface in the prescribed limits; the elements of the model of the fretting phenomenon in a pair of the ball part of the foot and insole are defined, which makes it possible to model study of the wear problem of movable contacting pairs, shows a method of calculation of the optimum level of the shock absorption of the human body with statically reel feet in phase of full support of the foot and presents a calculation method of the level of shock absorption created by the elastic spreading apart of the longitudinal arch, taken into account the pitch of the raise of the foot to the heel.

\section{References}

Alexandrov, S.P., Parshina, O.V. (1989). The study of stress-strain state of plantar muscles. Leather-shoe industry, 3, 25-32. 
Alexandrov, S.P., Zhukovskaya, T.V. (2013). Fretting in the system foot-shoe in phase of front shock and constructive prevention. News of higher educational institutions. Technology of consumer goods industry, 3, 64-66.

Gefen, A. (2002). Stress analysis of the standing foot following surgical plantar fascia release. Journal of Biomechanics, 35, 629-637.

Hessert, M.J., Vyas, M., Leach, J. (2005). Foot pressure distribution during walking in young and old adults. BMC Geriatrics, 5, 8.

Mruz, I.N. (2004). Design development of the shoes for the children with limb shortening. Extended abstract of candidate of technical sciences. Moscow.

Perepyolkin, A.I. (2013). Anatomy and function of the foot of the person at discontinuous load. Monograph. Moscow: Palmarium Academic Publishing.

Praet, S., Louwerens, J. (2003). The influence of shoe design on plantar pressures in neuropathic feet. Diabetes Care, 26(2), 441-445.

Ren-fei, G., Zhong-liang, Zh. (2006). To talk last design of comfortable shoe from ergonomics aspect. China Leather, 35(14), 119-121.

Rodgers, M. (1995). Dynamic foot biomechanics. Journal of Orthopaedic and Sports Physical Therapy, 21, 306-316.

Smirnova, L.M., Iuldashev, Z.M., Ioffe, R.Y., Ioffe, E.A. (2010). Computer podo-plantography on the software-hardware complex "Scan-M" at the screening examination of the preschool children. Medical devices, 2, 1-5. 